\title{
Wireless Patient Health Monitoring System
}

\author{
Manisha Shelar \\ R.G.P.V. University \\ Department of E \& T C \\ S.S.S.I.S.T. Sehore
}

\author{
Jaykaran Singh \\ R.G.P.V. University \\ Department of E \& T C \\ S.S.S.I.S.T. Sehore
}

\author{
Mukesh Tiwari \\ R.G.P.V. University \\ Department of E \& T C \\ S.S.S.I.S.T. Sehore
}

\begin{abstract}
This paper presents the development of a microcontroller based system for wireless heartbeat and temperature monitoring using ZigBee.In India many patients are dying because of heart attacks and reason behind that they are not getting timely and proper help. To give them timely and proper help first we want to continuous monitoring of patient health. The fixed monitoring system can be used only when the patient is on bed and this system are huge and only available in the hospitals in ICU.
\end{abstract}

The system is developed for home use by patients that are not in a critical condition but need to be constant or periodically monitored by clinician or family.[1] In any critical condition the SMS is send to the doctor or any family member.[2] So that we can easily save many lives by providing them quick service.

\section{INTRODUCTION}

Normally it is difficult to keep track on abnormalities in heartbeat count for patient itself manually. The average heartbeat per minute for 25-year old ranges between 140170 beats per minute while for a 60 -year old it is typically between 115-140 beats per minute and body temperature is 37degree Celsius or 98.6 Fahrenheit. Patients are not well versed with manual treatment which doctors normally use for tracking the count of heartbeat. So there must be some device which would help patient to keep track on their health by themselves. There are various instruments available in market to keep track on internal body changes. But there are many limitations regarding their maintenance due their heavy cost, size of instruments, and mobility of patients.
To overcome these limitations a device use to keep track on heartbeat count of patient should be easy to use, portable, light weighted, small size etc so that it give freedom of mobility for patient. The devices which can be carried everywhere to keep track on patient's health. This device that is a heartbeat sensor would help them to keep track on heartbeat counts of a patient and check for any abnormalities. If any varied change takes place it is notified. This notification would help to take an appropriate action at an instance of a time. This would save patients from the future health problem which would arise. This would also help patient's concern doctor to take an appropriate action at proper time.

\section{SYSTEM DESIGN}

The "Wireless patient monitoring system" is divided into two parts - Hardware \& Software. The hardware unit is further split into two units - Transmitter \& Receiver. The Transmitter unit is consisting of $\mu \mathrm{C}, \mathrm{ADC}$, Clock, Power Supply LCD, ZigBee modem etc.[4][6]

\subsection{Transmitter section}

The Transmitter will acquire values of physical parameters and will perform digital conversion of them for further processing. This digital data is then transmitted into air using ZigBee by the $\mu \mathrm{C}$. Thus it is not necessary to keep the board close to PC rather the transmitting unit can be placed at a far place within the range of ZigBee. The job of receiver unit is to receive those incoming values from air and to transfer into PC with the help of serial communication with COM Port.

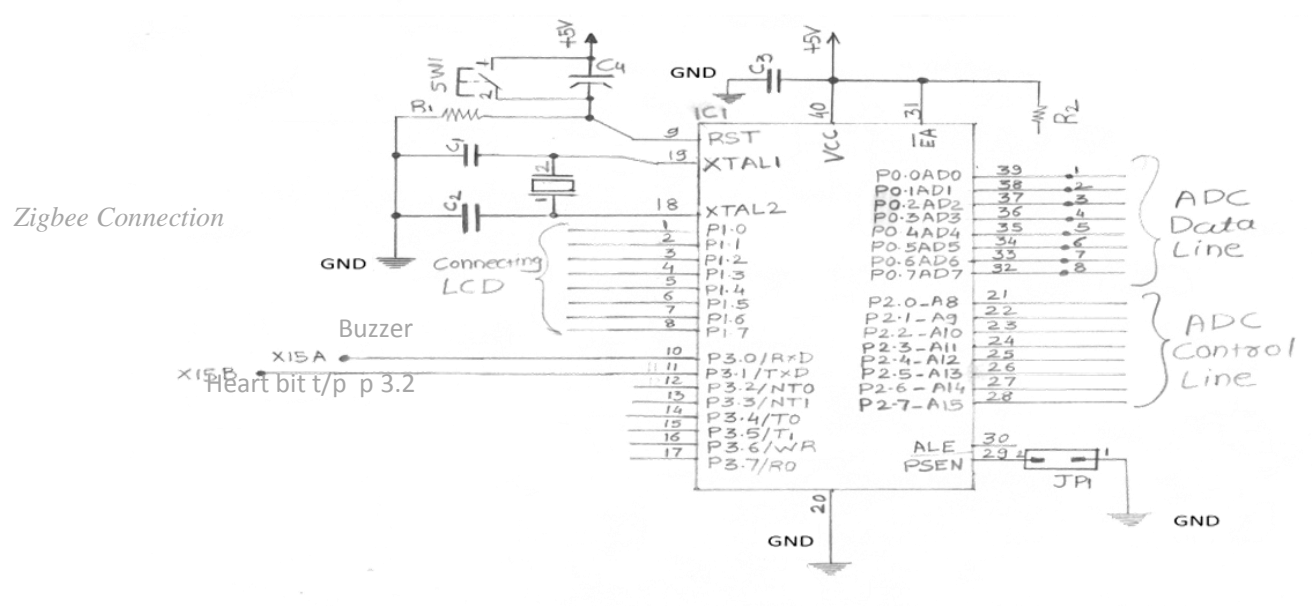

Figure 2.1 Circuit diagram of Microcontroller 


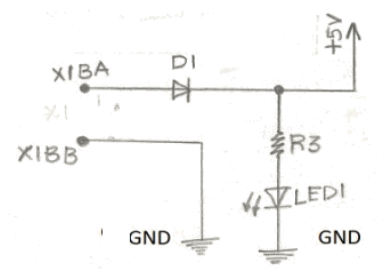

\subsection{Working of circuit}

\subsubsection{Heart Beat Monitor with microcontroller 8051}

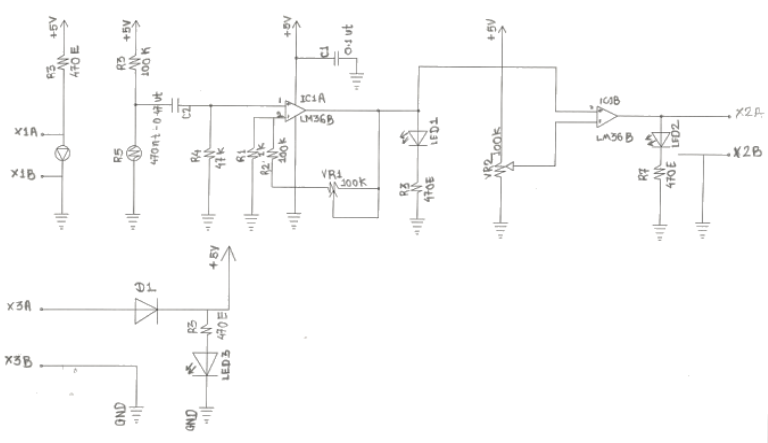

\section{Figure 2.2.1. Heart Beat Monitoring circuit using LM358}

The use of microcontroller is in every field even we can use it in the design and fabrication of biomedical equipments. A little example is here. The microcontroller at89s51 (8051) is here used to develop a heart beat monitoring system. By placing your finger in between a LED and photo resistance,

She/he can detect the pulses of heart, the analog voltages are further processed with an operational amplifier LM 358, this chip has two built in OPAMPs.[6] The TTL pulses or digital pulse are then feed to the external interrupt of microcontroller 8051. By using a software counter in the code, they can count the pulses, and the result the process is displayed on an LCD (2 line 16 characters).

$\mathrm{HB}=5184 / \mathrm{t}$

Where $\mathrm{t}$ is average of time delay between 2 consecutive pulses here use of first 5 pulses for calculation of HB.

The schematic circuit diagram of heart beat monitor is shown in Figure 2.2.1.

\subsubsection{Temperature sensor -The LM35}

The LM35 is an integrated circuit sensor that can be used to measure temperature with an electrical output proportional to the temperature (in ${ }^{\circ} \mathrm{C}$ ) .You can measure temperature more accurately than a using a thermistor. The sensor circuitry is sealed and not subject to oxidation, etc. The LM35 generates a higher output voltage than thermocouples and may not require that the output voltage be amplified.

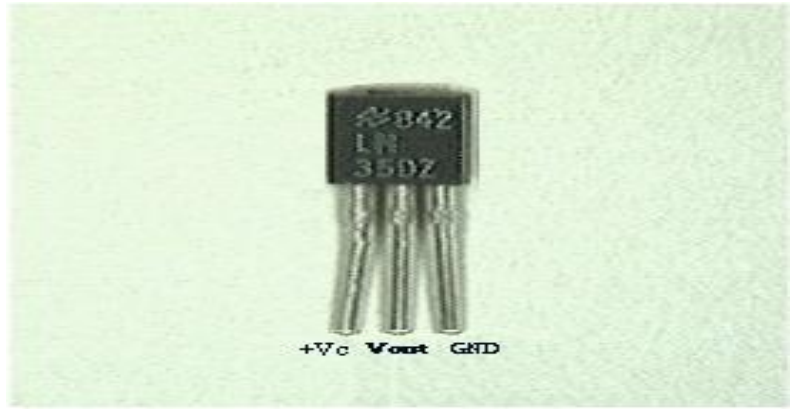

Figure 2.2.2. Temperature sensor -The LM35

It has an output voltage that is proportional to the Celsius temperature. The scale factor is $.01 \mathrm{~V} /{ }^{\circ} \mathrm{C}$ The $\mathrm{LM} 35$ does not require any external calibration or trimming and maintains an accuracy of $+/-0.4{ }^{\circ} \mathrm{C}$ at room temperature and $+/-0.8^{\circ} \mathrm{C}$ over a range of $0{ }^{\circ} \mathrm{C}$ to $+100{ }^{\circ} \mathrm{C}$.

\subsubsection{Working of $A D C$}

Micro controller sends the address of port to the ADC. For example 000 for channel $0 . \mu \mathrm{c}$ gives high pulse to ALE (Address Latch Enable) to latch the address into the ADC.It sends SOC (Start of Conversion) pulse, so that ADC starts performing successive appreciation for digital conversion on the selective channel. ADC starts processing the input channel for equivalent digital data. When the data is available on the port, it sends EOC (End of Conversion) pulse to the $\mu \mathrm{c} . \mu \mathrm{c}$ continuously monitors the EOC pulse. When it goes low, it takes the data from ADC. LM 555 is connected externally to the ADC in astable mode to give clock pulse.[6][7]

\subsection{4. $L C D 16 \times 2$}

It is called Liquid Crystal Display. There is a use of $16 \times 2$ characters LCD. This will be connected to microcontroller. The job of LCD will be to display all the system generated messages coming from the controller. LCD will provide interactive user interface.

\subsubsection{ZigBee}

ZigBee is a specification for a suite of high level communication protocols using small, low-power digital radios based on the IEEE 802.15.4-2003 standard for wireless personal area networks (WPANs), such as wireless headphones connecting with cell phones via shortrange radio.[3] The technology defined by the ZigBee specification is intended to be simpler and less expensive than other WPANs, such as Bluetooth. ZigBee is targeted at radio-frequency (RF) applications that require a low data rate, long battery life, and secure networking.

XBee and XBee-PRO DigiMesh 2.4 embedded RF modules provide wireless connectivity to end-point devices using a globally deployable $2.4 \mathrm{GHz}$ transceiver. These modules use the DigiMesh networking protocol. This innovative, peer-to-peer network offers users added network stability through self-healing, dense network operation and support for sleeping routers, extending the operational life of battery dependent networks.

XBee modules are ideal for low-power, low-cost applications. XBee-PRO modules are power-amplified versions of XBee modules for extended-range applications. Products in the XBee family are easy to use, share a common hardware footprint, and are fully interoperable 
with other XBee products utilizing the same technology. They are available in a variety of different protocols to suit different applications, enabling users to substitute one $\mathrm{XBee}$ module for another with minimal development time and risk.

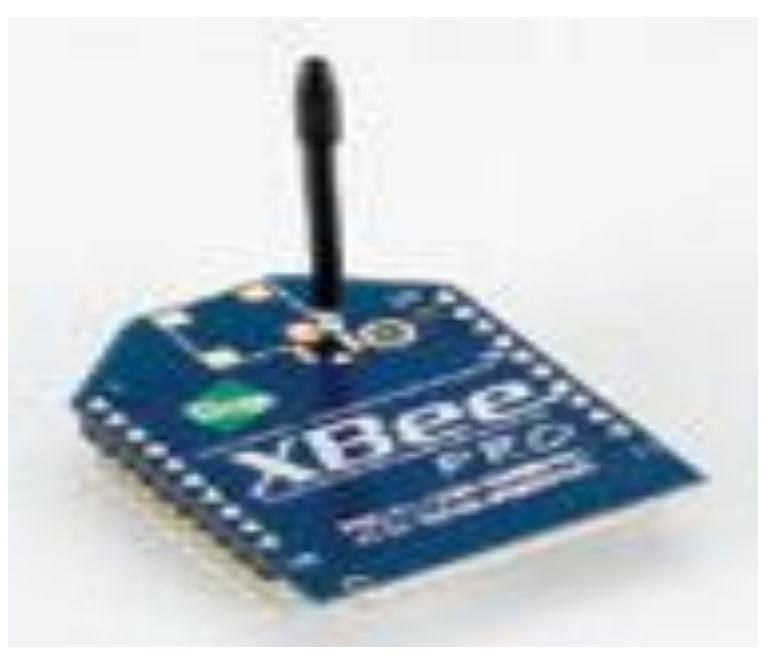

Figure 2.2.5. ZigBee Module

Digi's unsurpassed offering of Drop-in Networking products provide users with seamless communication between devices. XBee adapters deliver wireless connectivity to electronic devices in wired networks. XBee XTenders provide long-range backhaul and data conversion between $2.4 \mathrm{GHz}$ and $900 \mathrm{MHz}$. ConnectPort ${ }^{\mathrm{TM}}$ $\mathrm{X}$ gateways enable users to access and configure remote devices in a network.

Table 1. 2.4GHz Technology comparison.

\begin{tabular}{|c|c|c|c|c|}
\hline \multicolumn{5}{|c|}{$2.4 \mathrm{CHz}$ Technology Comparison } \\
\hline & Data Rate & $\begin{array}{l}\text { Number of } \\
\text { channels }\end{array}$ & $\begin{array}{l}\text { Interference } \\
\text { Avoidance } \\
\text { Method }\end{array}$ & $\begin{array}{l}\text { Minimum } \\
\text { Quiet } \\
\text { Bandwidth } \\
\text { Required }\end{array}$ \\
\hline $\begin{array}{c}\text { Wi-Fi } \\
(802.11 \mathrm{~b})\end{array}$ & 11 Mbps & 13 & $\begin{array}{l}\text { Fixed channel } \\
\text { collision } \\
\text { avoidance }\end{array}$ & $\begin{array}{l}22 \mathrm{MHz} \\
\text { (Static) }\end{array}$ \\
\hline Bluetooth & $723 \mathrm{Kbps}$ & 79 & $\begin{array}{l}\text { Adaptive } \\
\text { frequency } \\
\text { hopping }\end{array}$ & $\begin{array}{l}15 \mathrm{MHz} \\
\text { (Dynamic) }\end{array}$ \\
\hline WirelessUSB & $62.5 \mathrm{Kbps}$ & 79 & $\begin{array}{l}\text { Frequency } \\
\text { aglity }\end{array}$ & $\begin{array}{l}1 \mathrm{MHz} \\
\text { (Dynamic) }\end{array}$ \\
\hline Zigbee & $128 \mathrm{Kbps}$ & 16 & $\begin{array}{l}\text { Fixed channel } \\
\text { collision } \\
\text { avoidance }\end{array}$ & $3 \mathrm{MHz}$ (Static) \\
\hline
\end{tabular}

\section{CIRCUIT IMPLEMENTATION}

Initially, there is a development of the block diagram of system. Then developed circuits of individual blocks. All circuits are tested in lab and finalized. Next step is to develop PCB on pc. Printed circuit boards, or PCBs, form the core of electronic equipment domestic and industrial. Some of the areas where PCBs are intensively used are computers, process control, telecommunications and instrumentation.

\subsection{PCB designing}

\subsubsection{Eagle (Easily Applicable Graphical Layout Editor)}

The EAGLE is an editor, which is easy-to-use, yet powerful tool for designing printed circuit boards (PCBs). It is a complete platform for the development of any type of complicated \& sophisticated multilayered PCBs.

\subsubsection{PCB development}

There are many steps to develop PCB i.e.

- MANUFATCURING

- PANELISATION

- DRILLING

- PLATING

- ETCHING

- SOLDERMASK

- HOT AIR LEVELLING

After all this steps printed circuit board is manufactured and is now ready for the components to be soldered. After soldering and making connection of components using wires we got the model shown in figure 3.1.2

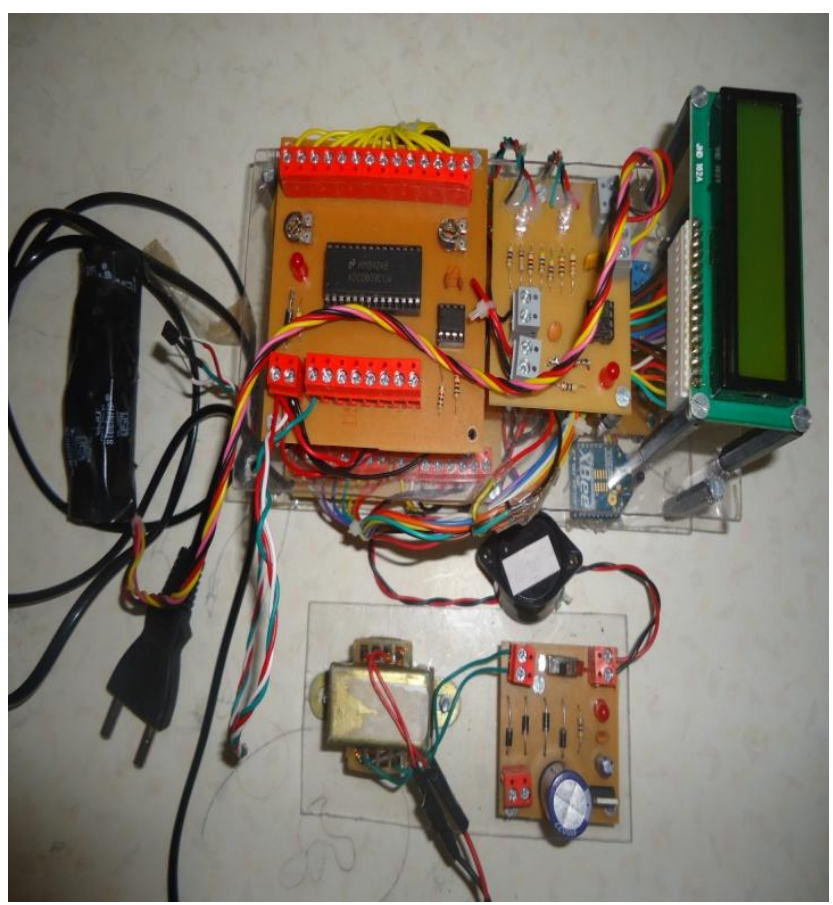

Figure.3.1.2 Implemented Circuit.

\section{RECEIVER SECTION}

The VB6 based software will display the data (Voltage values) of all channels in real time \& will store them into database for future reference.[9].The graphical and many 
more analysis on the stored data can be performed later on interactively.

The Software is also designed to monitor the values of those physical parameters so as they are always in the range of predefined limits i.e. Lower Limit \& Upper Limit. This can be achieved by monitoring the incoming data of each channel and by comparing it with both the limits. If the value does not reside within the range then the software will give command to pc \& message is sent on mobile connected to the parallel port. That will alert the doctor so that he can immediately take the necessary action to normalize the patient.

\section{RESULTS}

\subsection{Working condition of circuit}

When she/he can power on the circuit shown in the figure 4, all the LEDs on PCBs are glowing, indicating that circuit is working properly shown in figure 5 . Here there is a use of the industrial temperature sensor i.e. LM 35 which gives us room temperature in ${ }^{\mathbf{0}} \mathrm{C}$. That temperature is displayed on the LCD as shown in figure 5.1

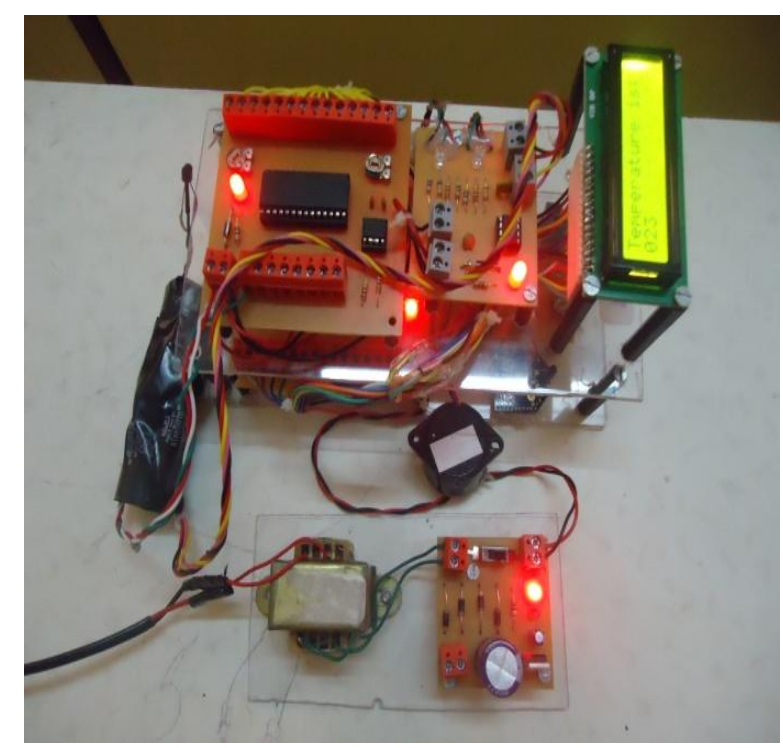

Figure 5 Implemented circuit in working condition

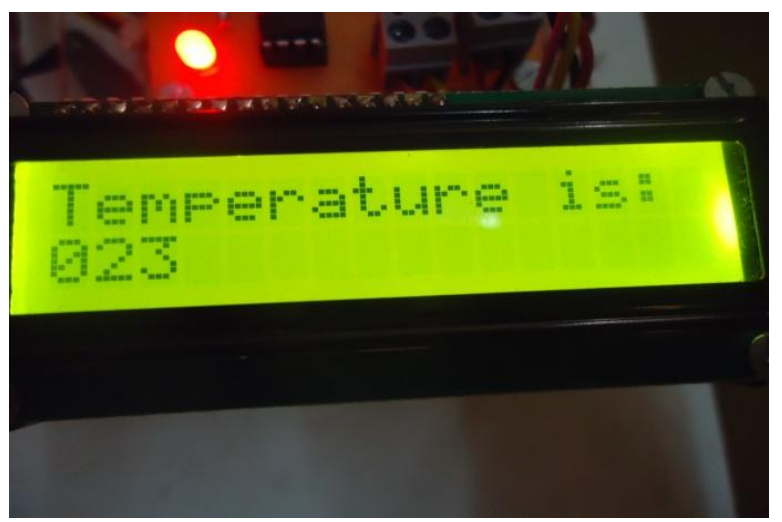

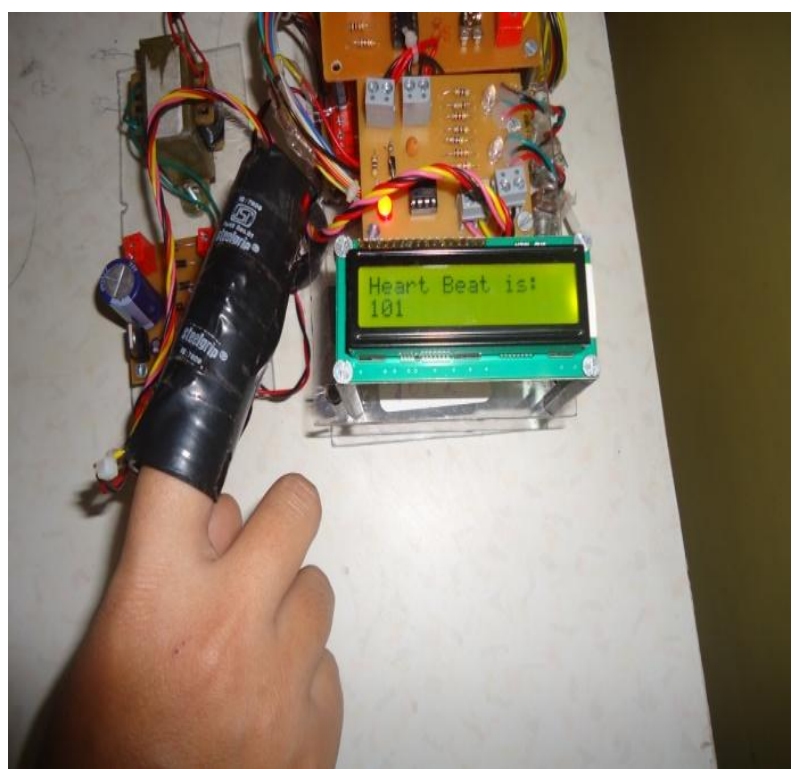

Figure 5.2 Heartbeat measurement

\subsection{Heartbeat measurement}

As shown in figure 5.2 there is a cavity for measurement of the heartbeat, which consist of a arrangement of LED and LDR. By placing your finger in between a LED and LDR, we can detect the pulses of heart, the analog voltages are further processed with an operational amplifier LM 358 , and this chip has two built in OPAMPs. Result is displayed on the LCD.This collected data is transmitted using ZigBee module. This data is received at the receiver section using same ZigBee module.

\subsection{Use of USB port of laptop}

Here there is a use of the laptop to show the results, so that ZigBee module is connected with the USB. Instead of using RS232 port of PC, use of the USB port of laptop.

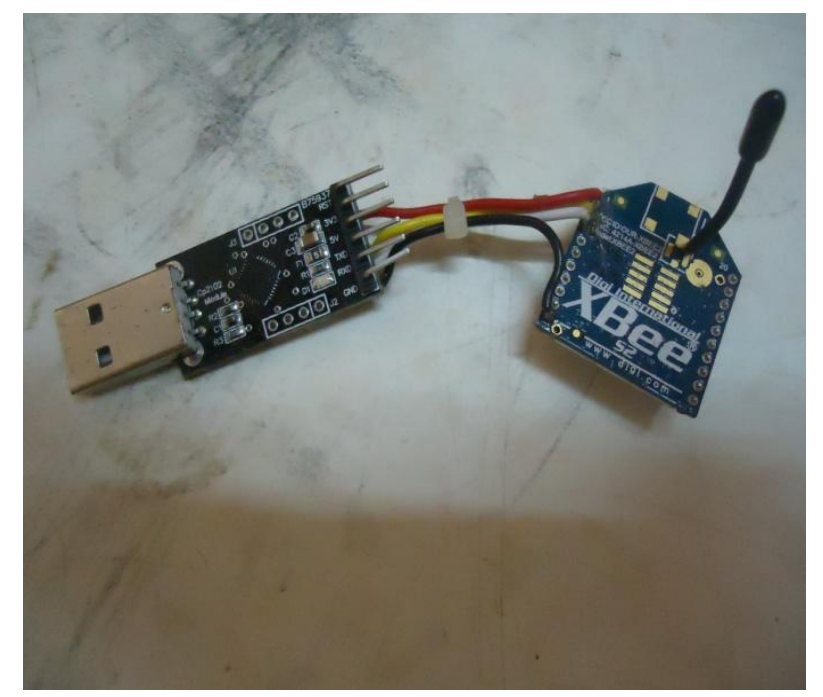

Figure 5.3 ZigBee module connection with USB

Figure 5.1 Temperature display on LCD 


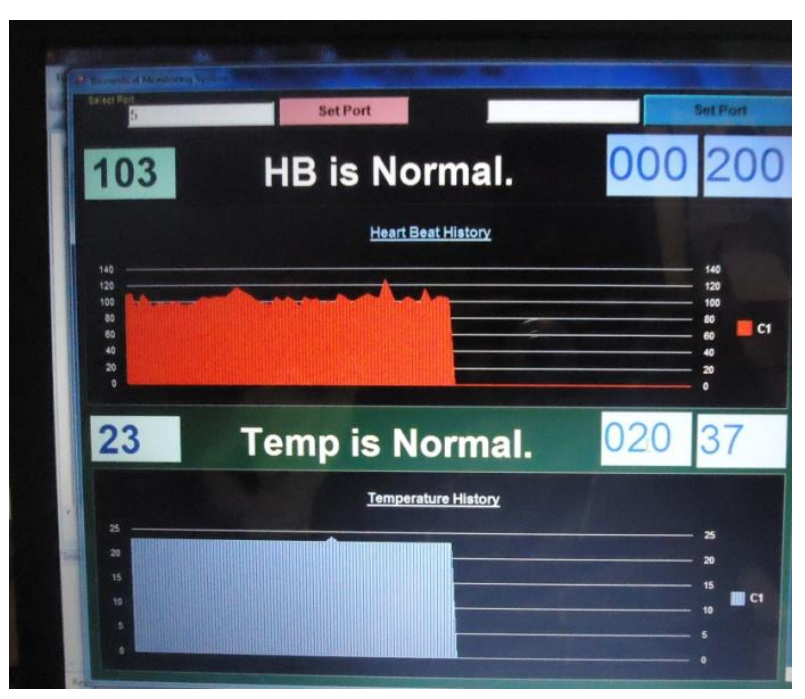

Figure 5.4 Graphical representation of Temp and HB

\subsection{Graphical representation of Temp and $\mathrm{HB}$}

The VB6 based software is used for programming. The programming of microcontroller is done in embedded C.The Figure 5.4 shows graphical representation of Heart beat and temperature. Doctor can set the lower and upper limits of both the parameter in run condition. If values of both the parameters are within the range then messages like "HB is normal" and "Temperature is normal "is displayed on the screen.Othetwise it will display the message like " $\mathrm{HB}$ is high" or " $\mathrm{HB}$ is Low" and message is send to the doctor mobile or any family member depends on the entered no .

\section{FUTURE DEVELOPMENT}

In addition to the system can also provide more than one numbers so that more than one user can receive emergency message.

According to availability of sensors or development in biomedical trend more parameter can be sense and monitor which will drastically improve the efficiency of the wireless monitoring system in biomedical field.

\section{CONCLUSION}

We have analyzed the wireless patient health monitoring system of temperature and heartbeat of humans using ZigBee, GSM, and SMS. Any abnormalities in health conditions are informed via SMS to the indicated mobile number through GSM. The hardware is implemented and the output is studied.

\section{REFERENCES}

[1] Ovidiu Apostu, Bogdan Hagiu, Sever Paşca, Wireless ECG Monitoring and Alarm System Using ZigBee"2011 The International Symposium on ADVANCED TOPIC INELECTRICAL ENGINEERING2068-7966/ATEE 2011.

[2] Warsuzarina Mat Jubadi, Siti Faridatul Aisyah Mohd ahak",Heartbeat Monitoring Alert via SMS", 978-1-4244-4683-4/09/\$25.00 @2009 IEEE.

[3] Goutam Motika, Abinash Prusty," Wireless Fetal Heartbeat Monitoring System Using ZigBee \& IEEE 802.15.4 Standard”, 2011 Second International Conference on Emerging Applications of Information Technology, 978-0-7695-4329-1/11 $\$ 26.00$ (C) 2011 IEEE DOI 10.1109/EAIT.2011.89

[4] Kenneth J. Ayala, The 8051 Microcontroller ARCHITECTURE, PROGRAMMING and APPLICATIONS, WEST PUBLISHING COMPANY, Page:131-197.

[5] Michael J. Pont, Embedded C, Edition 2002, Addison Wesley, Page: 57-87,217.

[6] Ramakant A. Gayakwad, Op-Amps and Linear Integrated Circuits, $4^{\text {th }}$ Edition, Prentice-Hall, Page:342, 417, 455.

[7] R.P.Jain, Digital Electronics, Tata McGraw-Hill

[8] Evangelos Petroutsos, Mark Ridgeway, Mastering Microsoft Visual Basic 2008, First Edition, Wiley Publishing, ISBN: 978-0-4701-8742-5.

[9] Dave Grundgeiger, Programming Visual Basic.Net, First Edition 2002, O'Reilly Publication, ISBN: 0596-00093-6, 464 Pages. 